\title{
CRESCIMENTO E NUTRIÇÃO DE MUDAS DE BANANEIRA EM SUBSTRATO CONTENDO RESÍDUOS DA AGROINDÚSTRIA DE CHÁ PRETO DURANTE A ACLIMATIZAÇÃO
}

\section{GROWTH AND NUTRITION OF BANANA SEEDLINGS IN SUBSTRATE CONTENT AGROINDUSTRY RESIDUES OF THE BLACK TEA DURING ACCLIMATIZATION}

\author{
Juliana Domingues LIMA ${ }^{1}$ \\ Silvia Helena MODENESE-GORLA DA SILVA ${ }^{2}$ \\ Eduardo Maciel HAITZMANN DOS SANTOS ${ }^{3}$ \\ Ana Paula de Souza LIMA $^{3}$ \\ Daiane Mitiko HIRATA ${ }^{3}$ \\ Franciele dos SANTOS ${ }^{3}$
}

\section{RESUMO}

O objetivo do trabalho foi avaliar a viabilidade da utilização de resíduo da agroindústria de chá preto como componente de substrato para aclimatização de mudas de bananeira micropropagadas. Foram instalados dois experimentos, no quais mudas da variedade Grand Naine foram aclimatizadas em terra de subsolo + casca de arroz carbonizada + Rendimax Floreira ${ }^{\circledR}$ ou terra de subsolo + casca de arroz carbonizada + resíduo de chá decomposto, na proporção 1:1:1 (v/v/v). No primeiro experimento, mudas cultivadas em substrato contendo resíduo decomposto de chá apresentaram menor crescimento quando comparadas com plantas cultivadas em substrato contendo Rendimax Floreira $\AA$, mesmo quando foi utilizado fertilizante $\mathrm{N}-\mathrm{P}_{2} \mathrm{O}_{5}-\mathrm{K}_{2} \mathrm{O}$ com liberação normal de nutrientes. No segundo experimento, quando se utilizou fertilizante de liberação controlada de nutrientes, não houve diferenças significativas na altura, diâmetro do colo, acúmulo de matéria seca e teores de macronutrientes na parte aérea entre as mudas cultivadas nos dois substratos.

Palavras-chave: Musa ssp.; micropropagação; produção de mudas.

\begin{abstract}
This work had for objective to evaluate the viability of the use of residues of the agroindustry of black tea as component of substrate for acclimatization of micropropagated banana seedlings. Two experiments were installed in which Grand Naine variety of seedlings were acclimatized in subsoil land + carbonized rice hull + commercial substrate Rendimax Floreira ${ }^{\circledR}$ or subsoil land + carbonized rice hull + residue of tea decomposed, in proportion 1:1:1 (v/v/v). In the first experiment, seedlings grown in the substrate containing decomposed residue of tea had lower growth when compared to plants grown on substrate containing Rendimax Floreira ${ }^{\circledR}$, even when $\mathrm{N}-\mathrm{P}_{2} \mathrm{O}_{5}-\mathrm{K}_{2} \mathrm{O}$ fertilizer was used with normal release of nutrients. In the second experiment, where fertilizer was used for controlled release of nutrients, there were no significant differences in height, base stem diameter, dry matter accumulation and levels of macronutrients in the shoots between seedlings grown in the two substrates.
\end{abstract}

Key-words: Musa ssp.; micropropagation; seedlings production.

\footnotetext{
'Engenheira Agrônoma, Campus Experimental de Registro, Universidade Estadual Paulista "Júlio de Mesquita Filho" (UNESP), Rua Rua Nélson Brihi Badur, n. 430, Vila Tupi, Registro - SP, Brasil. CEP 11.900-000. E-mail: judlima@registro.unesp.br.

${ }^{2}$ Tecnóloga em Processamento de Dados, Professora Assistente, Campus Experimental de Registro, Universidade Estadual Paulista “Júlio de Mesquita Filho”, Rua Rua Nélson Brihi Badur, n. 430, Vila Tupi, Registro - SP, Brasil. CEP 11.900-000. E-mail: silvia@registro.unesp.br.

${ }^{3}$ Graduandos do Curso de Agronomia do Campus Experimental de Registro, Universidade Estadual Paulista "Júlio de Mesquita Filho", Rua Rua Nélson Brihi Badur, n. 430, Vila Tupi, Registro - SP, Brasil. CEP 11.900-000. E-mail: eduhaitzmann@registro.unesp.br; anapaulaap@registro.unesp.br; daiane_mitiko@registro.unesp.br; franciele@registro.unesp.br.
} 


\section{INTRODUÇÃO}

Uma das tecnologias disponíveis para o produtor de banana é o uso de mudas micropropagadas que apresentam como principais vantagens o fato de serem livres de patógenos e pragas, o que reduz a dispersão de organismos fitoparasitas (Damasco et al., 1996), além de possuírem identidade genética garantida. Como desvantagem, torna-se necessário um período de aclimatização em viveiro sombreado em recipientes contendo substrato que propicie um rápido crescimento inicial das mudas antes do plantio no campo (Silva et al., 1999).

O substrato tem papel fundamental na produção de mudas de qualidade e exerce influência marcante na arquitetura do sistema radicular, no estado nutricional das plantas (Vale et al., 2004) e na translocação de água no sistema solo-plantaatmosfera (Maciel et al., 2000).

As condições ideais de um substrato dependem da faixa de exigência das espécies cultivadas e dificilmente se encontra um material que propicie todas as condições necessárias para o crescimento das plantas (Grolli, 1991). Além disso, os materiais disponíveis, em geral, apresentam uma série de problemas para as plantas em virtude de possuírem características muito diversas (Bordas et al., 1988), e por isso, normalmente os substratos são constituídos de mais de um material, em geral solo e matéria orgânica (Backes, 1988), sendo que a matéria orgânica é a fração mais importante do substrato (Miller \& Jones, 1995). Na escolha dos componentes do substrato também deve ser observado o custo e a disponibilidade dos materiais.

De modo geral, resíduos agroindustriais vêm sendo progressivamente utilizados como alternativa para minimizar o impacto ambiental provocado por tais resíduos sólidos. Uma das formas de sua utilização é na composição de substratos agrícolas para produção de mudas como demonstrado em diversos trabalhos de pesquisa (Morgado et al., 2000; Serrano et al., 2006; Resende et al., 2005; Correia et al., 2003).

$\mathrm{Na}$ região do Vale do Ribeira, as indústrias de chá preto (obtido a partir da oxidação de folhas jovens de Camellia sinensis L.) são geradoras de resíduos que podem poluir o ambiente, mas que por outro lado, são passíveis de serem reciclados.

Pelo exposto objetivou-se com o presente trabalho avaliar a eficiência do uso do resíduo decomposto da agroindústria de chá preto como substrato para aclimatização de mudas de bananeira micropropagadas.

\section{MATERIAL E MÉTODOS}

Os experimentos foram conduzidos no Campus Experimental de Registro, UNESP, localizado no município de Registro, SP (latitude $24^{\circ} 29^{\prime} 22^{\prime \prime} \mathrm{S}$, longitude 4750'10" W, altitude de 11,99 $\mathrm{m})$, que possui clima tropical úmido Af (Köeppen), com transição para Cfa, sem estação seca definida, sob telado com $50 \%$ de sombreamento, conforme recomendado por Scaranari (2006).

O resíduo de chá foi obtido na empresa Yamatea, localizada no município de Pariquera-Açu, $\mathrm{SP}$ e as mudas de bananeira da cultivar Grand Naine, obtidas por meio de micropropagação de ápices caulinares sem bainha foliar, no laboratório BIOVALE da Agência Paulista de Agronegócios (APTA), localizado no mesmo município.

O primeiro experimento foi realizado no delineamento inteiramente casualizado, em esquema fatorial $2 \times 2$ dduas misturas de substratos [S1 - terra de subsolo + casca de arroz carbonizada + Rendimax Floreira ${ }^{\circledR}(1: 1: 1 ; \mathrm{v} / \mathrm{v} / \mathrm{v})$ e $\mathrm{S} 2$ - terra de subsolo + casca de arroz carbonizada + resíduo de chá decomposto $(1: 1: 1 ; \mathrm{v} / \mathrm{v} / \mathrm{v})$ ] e dois níveis de adubação [ausência e fertilizante de liberação normal de nutrientes]\}, com 10 repetições.

Após o preparo dos substratos reunindo os diferentes componentes, foram coletadas amostras para análise química as quais foram realizadas no laboratório da Faculdade de Ciências Agronômicas, Botucatu, UNESP, cujos resultados se encontram na Tabela 1.

O plantio foi feito em vasos contendo $3,5 \mathrm{dm}^{3}$ de cada um dos substratos, utilizando mudas de raiz nua, com cerca de $5 \mathrm{~cm}$ de altura, 4 a 5 folhas e em média 6 raízes adventícias com comprimento médio de $2,53 \mathrm{~cm}$.

Aos 21 dias após o plantio, metade dos vasos contendo cada um das misturas de substratos recebeu adubação auxiliar em cobertura na dose de $10 \mathrm{~g}_{\text {planta-1 }}{ }^{-1}$ da fórmula $10-10-10$ de $\mathrm{N}$ $\mathrm{P}_{2} \mathrm{O}_{5}-\mathrm{K}_{2} \mathrm{O}$, com liberação normal de nutrientes. Realizou-se diariamente a irrigação de maneira uniforme com cerca $0,1 \mathrm{dm}^{3}$ de água de torneira em todos os vasos. Semanalmente, foram avaliadas as características de crescimento, altura, determinada pela medida desde o coleto até a inserção da primeira folha totalmente expandida, no sentido da base para o ápice, e o diâmetro do colo.

O segundo experimento foi realizado a partir da análise dos resultados obtidos no primeiro, sendo instalado no delineamento experimental inteiramente casualizado em esquema fatorial $2 \times 3$ \{duas misturas de substratos [S1 - terra de subsolo + casca de arroz carbonizada + Rendimax Floreira ${ }^{\circledR}$ $(1: 1: 1 ; \mathrm{v} / \mathrm{v} / \mathrm{v})$ e S2 - terra de subsolo + casca de arroz carbonizada + resíduo de chá decomposto $(1: 1: 1 ; \mathrm{v} /$ $\mathrm{v} / \mathrm{v})$ ] e três formas de adubação [SF - sem fertilizante, FLNN - fertilizante formulado 14-14-14 de N-P $\mathrm{O}_{5}$ $\mathrm{K}_{2} \mathrm{O}$ com liberação normal de nutrientes e FLCN ${ }^{2}$ fertilizante 14-14-14 com liberação controlada de nutrientes em 3 a 4 meses (Osmocote $®$ )]\}, com 10 repetições. Os dois fertilizantes foram aplicados na dose de $10 \mathrm{~g} \mathrm{planta}^{-1}$, sendo o primeiro, em cobertura aos 21 dias após o plantio, e o segundo, adicionado no momento da mistura dos componentes dos substratos antes do plantio.

O experimento foi avaliado semanalmente, por meio da altura e do diâmetro do colo das mudas. Ao final do experimento, foi determinada a área foliar e a matéria seca acumulada na raiz e na parte aérea, e a área foliar das plantas. A área foliar foi obtida por meio do medidor de área foliar LI-3100® e a matéria 
LIMA, J.D. et al. Crescimento e nutrição de mudas de bananeira...

TABELA 1 - Características e composição química média dos substratos utilizados no experimento antes da adubação química.

\begin{tabular}{|c|c|c|}
\hline & Substrato 1 & Substrato 2 \\
\hline & \multicolumn{2}{|c|}{$\%$} \\
\hline Umidade & 30,9 & 26,7 \\
\hline Matéria Orgânica & 25 & 28 \\
\hline $\mathrm{C} / \mathrm{N}$ & $13 / 1$ & $28 / 1$ \\
\hline \multirow[t]{2}{*}{$\mathrm{pH}$} & 5,21 & 5,05 \\
\hline & \multicolumn{2}{|c|}{$\mathrm{g} \mathrm{kg}^{-1} \mathrm{MS}$} \\
\hline $\mathrm{N}$ & 10,8 & 5,5 \\
\hline $\mathrm{P}_{2} \mathrm{O}_{5}$ & 2,4 & 2,6 \\
\hline $\mathrm{K}_{2} \mathrm{O}$ & 2,9 & 1,6 \\
\hline $\mathrm{C}$ & 139 & 156 \\
\hline $\mathrm{Ca}$ & 2,4 & 3,2 \\
\hline $\mathrm{Mg}$ & 1,3 & 4,0 \\
\hline \multirow[t]{2}{*}{$\mathrm{S}$} & 2,7 & 2,2 \\
\hline & \multicolumn{2}{|c|}{$\mathrm{mg} \mathrm{kg}^{-1} \mathrm{MS}$} \\
\hline $\mathrm{Na}$ & 300 & 300 \\
\hline $\mathrm{Cu}$ & 60 & 58 \\
\hline $\mathrm{Fe}$ & 42000 & 36000 \\
\hline $\mathrm{Mn}$ & 110 & 142 \\
\hline $\mathrm{Zn}$ & 80 & 70 \\
\hline
\end{tabular}

Substrato 1 - terra de subsolo + casca de arroz carbonizada + Rendimax Floreira $(1: 1: 1 ; \mathrm{v} / \mathrm{v} / \mathrm{v})$;

Substrato 2 - terra de subsolo + casca de arroz carbonizada + resíduo de chá decomposto (1:1:1; v/v/v).

seca, a pós a secagem da parte aérea e das raízes, em estufa a $65^{\circ} \mathrm{C}$ até atingir peso constante. Após a determinação da matéria seca de ambas as partes em balança analítica, uma porção da parte aérea foi triturada em moinho tipo Willey e encaminhada para - laboratório da Faculdade de Ciências Agronômicas, Botucatu, UNESP, para determinação dos teores de macronutrientes.

Os dados obtidos foram submetidos à análise de variância pelo teste $F$ e quando significativo, comparou-se às médias de cada tratamento por meio do teste Tukey a $5 \%$ de probabilidade.

\section{RESULTADOS E DISCUSSÃO}

No primeiro experimento, mudas cultivadas no substrato 1 , aos 56 dias após o plantio, apresentaram crescimento relativamente superior em altura e diâmetro do colo, quando comparadas com mudas cultivadas com substrato 2 contendo resíduo de chá decomposto (Tabela 2). Estas diferenças podem estar relacionadas ao fato do Rendimax Floreira ${ }^{\circledR}$, constituído basicamente de vermiculita e casca de Pinus moída, utilizado na composição do substrato 2 , apresentar uma concentração relativamente maior de nitrogênio e potássio, essenciais para o crescimento das plantas, quando comparado ao resíduo decomposto de chá (Tabela 1). Além disso, a relação $\mathrm{C} / \mathrm{N}$ do substrato 2 contendo resíduo decomposto de chá foi mais elevada (28/1), quando comparada com o substrato 1 contendo Rendimax Floreira ${ }^{\circledR}(13 / 1)$, indicando que no substrato 1 havia pronta disponibilidade do nitrogênio.

Com relação ao segundo experimento, não se constatou diferenças no crescimento final seja em altura ou em diâmetro do colo entre mudas cultivadas no substrato 1 e substrato 2 com adição do fertilizante de liberação controlada de nutrientes (Tabelas 3 e 4). No entanto, quando o substrato 2 recebeu fertilizante de liberação normal de nutrientes, o crescimento em altura e em diâmetro do colo foi inferior ao observado nas mudas cultivadas no Substrato 1 e que receberam a mesma adubação. Sem adubação, as mudas cultivadas no substrato 1 exibiram crescimento superior em altura e diâmetro do colo, em relação às cultivadas com substrato 2 .

Para área foliar, quando foi utilizado fertilizante de liberação controlada de nutrientes, mudas cultivadas no substrato 2 não diferiram das cultivadas no substrato 1 (Tabela 5 ). Para matéria seca da parte aérea, ocorreu resposta semelhante à observada para área foliar (Tabela 6). Todavia, os efeitos dos dois fertilizantes e dos substratos utilizados na acumulação de matéria seca da raiz foram apenas significativos quando isolados, ainda assim, demonstrando haver maior acumulação com o uso de fertilizante de liberação controlada, independente do substrato.

Segundo Kozlowski et al. (1991), mudas com maior área foliar na época de serem levadas para o campo apresentam crescimento inicial mais rápido, em virtude da maior produção de fotoassimilados e posterior alocação para outras partes da planta (drenos). Contudo, Morgado (1998), considera a matéria seca da parte aérea, como uma boa indicadora da capacidade de resistência das mudas às condições adversas após o plantio no campo. 
LIMA, J.D. et al. Crescimento e nutrição de mudas de bananeira...

TABELA 2 - Valores médios de altura $(\mathrm{cm})$ e diâmetro do colo $(\mathrm{cm})$ de mudas de bananeira em função do substrato e da adubação, aos 56 dias após o transplante. SF (sem fertilizante); FLNN (fertilizante de liberação normal de nutrientes); Substrato 1 (terra de subsolo + casca de arroz carbonizada + Rendimax Floreira ${ }^{\circledR}$ ); Substrato 2 (terra de subsolo + casca de arroz carbonizada + resíduo de chá decomposto).

\begin{tabular}{|c|c|c|c|c|}
\hline & & & & colo \\
\hline & SF & FLNN & SF & FLNN \\
\hline Rendimax & $7,07 \mathrm{Aa}$ & $11,21 \mathrm{Ab}$ & $1,49 \mathrm{Aa}$ & $2,03 \mathrm{Ab}$ \\
\hline Resíduo de chá & $3,29 \mathrm{Ba}$ & $8,79 \mathrm{Bb}$ & $0,98 \mathrm{Ba}$ & $1,61 \mathrm{Bb}$ \\
\hline $\mathrm{CV}(\%)$ & & & & \\
\hline
\end{tabular}

Médias seguidas de mesmas letras, maiúsculas na coluna e minúsculas na linha, não diferem entre si ao nível de $5 \%$ de probabilidade pelo teste de Tukey.

TABELA 3 - Altura (cm) de mudas de bananeira 'Grand Nine' em função da forma de fertilizante e do substrato, aos 56 dias após o transplante. SF (sem fertilizante); FLNN (fertilizante de liberação normal de nutrientes); FLCN (fertilizante de liberação controlada de nutrientes); Substrato 1 (terra de subsolo + casca de arroz carbonizada + Rendimax Floreira ${ }^{\circledR}$ ); Substrato 2 (terra de subsolo + casca de arroz carbonizada + resíduo de chá decomposto).

\begin{tabular}{lllll}
\hline & SF & FLNN & FLCN & Médias \\
\hline Substrato 1 & $10,36 \mathrm{~A} \mathrm{c}$ & $16,82 \mathrm{~A} \mathrm{~b}$ & $21,83 \mathrm{~A} \mathrm{a}$ & $16,33 \mathrm{~A}$ \\
Substrato 2 & $7,24 \mathrm{~B} \mathrm{c}$ & $13,27 \mathrm{~B} \mathrm{~b}$ & $19,96 \mathrm{~A} \mathrm{a}$ & $13,49 \mathrm{~B}$ \\
\hline Médias & $8,80 \mathrm{c}$ & $15,04 \mathrm{~b}$ & $20,89 \mathrm{a}$ &
\end{tabular}

Médias seguidas da mesma letra maiúscula na coluna e da mesma letra minúscula na linha, não diferem entre si, a $5 \%$ de probabilidade pelo teste Tukey.

TABELA 4 - Diâmetro do colo (cm) de mudas de bananeira 'Grand Nine' em função da forma de fertilizante e do substrato, aos 56 dias após o transplante. SF (sem fertilizante); FLNN (fertilizante de liberação normal de nutrientes); FLCN (fertilizante de liberação controlada de nutrientes); Substrato 1 (terra de subsolo + casca de arroz carbonizada + Rendimax Floreira ${ }^{\circledR}$ ); Substrato 2 (terra de subsolo + casca de arroz carbonizada + resíduo de chá decomposto).

\begin{tabular}{lllll}
\hline & SF & FLNN & FLCN & Médias \\
\hline Substrato 1 & $1,35 \mathrm{~A} \mathrm{c}$ & $2,21 \mathrm{~A} \mathrm{~b}$ & $2,65 \mathrm{~A} \mathrm{a}$ & $2,07 \mathrm{~A}$ \\
Substrato 2 & $1,07 \mathrm{~B} \mathrm{c}$ & $1,75 \mathrm{~B} \mathrm{~b}$ & $2,45 \mathrm{~A} \mathrm{a}$ & $1,75 \mathrm{~B}$ \\
\hline Médias & $1,21 \mathrm{c}$ & $1,98 \mathrm{~b}$ & $2,55 \mathrm{a}$ &
\end{tabular}

Médias seguidas da mesma letra maiúscula na coluna e da mesma letra minúscula na linha, não diferem entre si, a $5 \%$ de probabilidade pelo teste Tukey.

TABELA 5 - Área foliar $\left(\mathrm{cm}^{2}\right)$ de mudas de bananeira 'Grand Nine' em função da forma de fertilizante e do substrato, aos 56 dias após o transplante. SF (sem fertilizante); FLNN (fertilizante de liberação normal de nutrientes); FLCN (fertilizante de liberação controlada de nutrientes); Substrato 1 (terra de subsolo + casca de arroz carbonizada + Rendimax Floreira ${ }^{\text {) }}$; Substrato 2 (terra de subsolo + casca de arroz carbonizada + resíduo de chá decomposto).

\begin{tabular}{lllll}
\hline & \multicolumn{1}{c}{ SF } & FLNN & FLCN & Médias \\
\hline Substrato 1 & $346,50 \mathrm{~A} \mathrm{c}$ & $1159,01 \mathrm{~A} \mathrm{~b}$ & $1500,69 \mathrm{~A}$ a & $1002,06 \mathrm{~A}$ \\
Substrato 2 & $215,17 \mathrm{~B} \mathrm{c}$ & $693,17 \mathrm{~B} \mathrm{~b}$ & $1460,00 \mathrm{~A} \mathrm{a}$ & $789,44 \mathrm{~B}$ \\
\hline Médias & $280,83 \mathrm{c}$ & $926,08 \mathrm{~b}$ & $1480,35 \mathrm{a}$ &
\end{tabular}

Médias seguidas da mesma letra maiúscula na coluna e da mesma letra minúscula na linha, não diferem entre si, a $5 \%$ de probabilidade pelo teste Tukey.

O crescimento em altura no final do experimento observado nas plantas cultivadas nos dois substratos e com a adição fertilizante de liberação controlada de nutrientes foi similar ao observado por Scaranari (2006) para mudas de bananeira da mesma variedade, aclimatadas no substrato Potato $囚$. No entanto, para área foliar, matéria seca da raiz e da parte aérea, os resultados do presente estudo foram superiores aos encontrados pelo mesmo autor. 
TABELA 6 - Massa seca (g) da parte aérea (MSPA) e da raiz (MSR) de mudas de bananeira 'Grand Nine' em função da forma de fertilizante e do substrato, aos 56 dias após o transplante. SF (sem fertilizante); FLNN (fertilizante de liberação normal de nutrientes); FLCN (fertilizante de liberação controlada de nutrientes); Substrato 1 (terra de subsolo + casca de arroz carbonizada + Rendimax Floreira ${ }^{\circledR}$ ); Substrato 2 (terra de subsolo + casca de arroz carbonizada + resíduo de chá decomposto).

\begin{tabular}{lcccccccc}
\hline & \multicolumn{2}{c}{ SF } & \multicolumn{2}{c}{ FLNN } & \multicolumn{2}{c}{ FLCN } & \multicolumn{2}{c}{ Médias } \\
\hline & MSPA & MSR & MSPA & MSR & MSPA & MSR & MSPA & MSR \\
\hline Rendimax & $5,43 \mathrm{~A} \mathrm{c}$ & - & $6,96 \mathrm{~A} \mathrm{~b}$ & - & $11,48 \mathrm{a} \mathrm{A}$ & - & $7,99 \mathrm{~A}$ & $3,46 \mathrm{~A}$ \\
Resíduo & $3,28 \mathrm{~B} \mathrm{c}$ & - & $5,52 \mathrm{~B} \mathrm{~b}$ & - & $11,29 \mathrm{a} \mathrm{A}$ & - & $6,69 \mathrm{~B}$ & $3,12 \mathrm{~B}$ \\
\hline Médias & $4,35 \mathrm{c}$ & $2,58 \mathrm{c}$ & $6,24 \mathrm{~b}$ & $3,40 \mathrm{~b}$ & $11,44 \mathrm{a}$ & $3,89 \mathrm{a}$ & &
\end{tabular}

Médias seguidas da mesma letra maiúscula na coluna e da mesma letra minúscula na linha, não diferem entre si, a $5 \%$ de probabilidade pelo teste Tukey.

As diferenças no crescimento das mudas que receberam fertilizante com liberação normal e controlada de nutrientes podem ser explicadas pela disponibilidade contínua dos nutrientes minimizando, assim, a probabilidade de ocorrer deficiências nutricionais durante o período de aclimatização das mudas em relação à utilização de fertilizantes solúveis com liberação normal de nutrientes. Além disso, segundo Huett (1997), outros benefícios da utilização deste tipo de fertilizante em relação à utilização de fertilizantes solúveis é a baixa perda de nutrientes por lixiviação, menor possibilidade danos causados pela salinidade nas mudas (Sharma, 1979), além da economia de mão-de-obra, uma vez que o fertilizante é adicionado ao substrato no momento do seu preparo sem a necessidade de parcelamento. Como principal desvantagem, os fertilizantes de liberação lenta por serem produtos importados apresentam custo superior às fontes solúveis (Scivittaro, 2004).

TABELA 7 - Teores de macronutrientes ( $\mathrm{g} \mathrm{kg}^{-1} \mathrm{MS}$ ) na parte aérea de mudas de bananeira 'Grand Nine' em função da forma de fertilizante utilizada e do substrato, aos 56 dias após o transplante. SF (sem fertilizante); FLNN (fertilizante de liberação normal de nutrientes); FLCN (fertilizante de liberação controlada de nutrientes); Substrato 1 (terra de subsolo + casca de arroz carbonizada + Rendimax Floreira ${ }^{\circledR}$ ); Substrato 2 (terra de subsolo + casca de arroz carbonizada + resíduo de chá decomposto).

\begin{tabular}{|c|c|c|c|c|c|c|}
\hline & \multicolumn{2}{|c|}{ SF } & \multicolumn{2}{|c|}{ FLNN } & \multicolumn{2}{|c|}{ FLCN } \\
\hline & Substrato 1 & Substrato 2 & Substrato 1 & Substrato 2 & Substrato 1 & Substrato 2 \\
\hline $\mathrm{N}$ & $19,2 \mathrm{a}$ & $13,4 \mathrm{~b}$ & $27,3 a$ & $21,4 \mathrm{~b}$ & $32,1 \mathrm{a}$ & $30,3 a$ \\
\hline $\mathrm{P}$ & $1,9 \mathrm{~b}$ & $2,6 a$ & $2,4 b$ & $3,1 \mathrm{a}$ & $3,8 \mathrm{a}$ & $4,0 \mathrm{a}$ \\
\hline $\mathrm{K}$ & $16,3 \mathrm{a}$ & $10,2 b$ & 20,5 a & $14,6 \mathrm{~b}$ & 29,3 a & 27,6 a \\
\hline $\mathrm{Ca}$ & $4,9 \mathrm{a}$ & $4,1 \mathrm{a}$ & $5,2 \mathrm{a}$ & $5,7 \mathrm{a}$ & $6,1 \mathrm{a}$ & $6,9 a$ \\
\hline $\mathrm{Mg}$ & $4,0 \mathrm{a}$ & $3,7 \mathrm{a}$ & $4,2 \mathrm{a}$ & $4,5 \mathrm{a}$ & $4,3 \mathrm{a}$ & $4,8 \mathrm{a}$ \\
\hline$S$ & $1,3 a$ & $0,9 \mathrm{~b}$ & $2 a$ & $1,9 a$ & $2,3 a$ & $2,1 \mathrm{a}$ \\
\hline
\end{tabular}

Médias seguidas da mesma letra na linha, para determinada forma de adubação, não diferem entre si, a $5 \%$ de probabilidade pelo teste Tukey.

Outros trabalhos também mostraram o efeito benéfico do uso do Osmocote®. Oliveira et al. (1995) avaliaram o efeito de diferentes doses de Osmocote $\AA$ (17-09-13) adicionado ao substrato comercial Plantmax® na produção de mudas de cafeeiro e concluíram que aquelas que receberam o Osmocote $\AA$ apresentaram maior altura, vigor, qualidade e sanidade, além da antecipação de 40 dias na liberação das mesmas e economia de mão de obra. Andrade Neto et al. (1999) verificaram também em mudas de cafeeiro que o maior crescimento foi obtido quando ao esterco de curral foi adicionado Osmocote ${ }^{\circledR}$.

A maior concentração de macronutrientes na parte aérea das mudas de bananeira aos 56 dias após o plantio foi observada para $\mathrm{N}, \mathrm{K}$ e $\mathrm{Ca}$ independente do substrato e do tipo de fertilizante utilizado refletiram os resultados da análise química do substrato e o tipo de fertilizante utilizado (Tabela 7), sendo próximos aos encontrados por Sousa et al. (2000) e mais baixos na ausência de fertilização. Com a utilização do fertilizante de liberação normal de nutrientes, os teores de nitrogênio e potássio em mudas cultivadas no substrato 1 foram superiores aos encontrados para mudas no substrato 2 . Todavia, quando foi utilizado fertilizante de liberação controlada de nutrientes, as diferenças tornaram-se não significativas. Assim, evidenciase que a defasagem no crescimento entre mudas cultivadas no substrato 1 e substrato 2 foi devida ao menor teor de $\mathrm{N}$ e $\mathrm{K}$ no resíduo de chá, podendo ser superada com a utilização do fertilizante de liberação controlada de nutrientes. Apenas para o teor de fósforo na parte 
LIMA, J.D. et al. Crescimento e nutrição de mudas de bananeira...

aérea das mudas cultivadas no substrato 2 , sem adubação e com fertilizante de liberação normal de nutrientes, os valores obtidos foram superiores aos de plantas cultivadas no substrato 1 , na mesma condição (Tabela 7). Esses resultados podem ser atribuídos ao efeito de concentração, conforme também observado por Seabra Filho (1994) e Sousa et al. (2000) em mudas de bananeira, indicando que o menor crescimento das plantas nesse substrato acarretou acúmulo de fósforo na matéria seca.

A adoção do substrato 2 reduz o gasto com mão-de-obra e aquisição do substrato comercial, que supera o custo 3,6 vezes mais elevado da utilização do fertilizante de liberação controlada de nutrientes em relação ao fertilizante de liberação normal de nutrientes, permitindo a aclimatização econômica das mudas de bananeira e a destinação mais adequada do resíduo da agroindústria do chá preto.

\section{CONCLUSÃO}

A aclimatização de mudas de bananeira pode ser realizada com sucesso e economia, utilizando-se o substrato composto por terra de subsolo + casca de arroz carbonizada + resíduo de chá decomposto, adicionando-se fertilizante de liberação controlada de nutrientes.

\section{REFERÊNCIAS}

1. ANDRADE NETO, A.; MENDES, A. N. G.; GUIMARÃES, P. T. G. Avaliação de substratos alternativos e tipos de adubação para a produção de mudas de cafeeiro (Coffea arabica L.) em tubetes. Ciência e Agrotecnologia, $v$. 23, n. 2, p. 270-280, 1999.

2. BACKES, M. A. Composto de lixo urbano como substrato para plantas ornamentais. 1988.80 p. Dissertação (Mestrado em Fitotecnia) - Universidade Federal do Rio Grande do Sul, Porto Alegre, 1988.

3. BORDAS, J. M. C.; BACKES, M. A.; KÄMPF, A. N. Características físicas e químicas de substratos comerciais. In: CONGRESSO FLORESTAL ESTADUAL, 6., Nova Prata, 1988. Anais... Nova Prata: Secretaria da Agricultura do Rio Grande do Sul, 1988. v. 1, p. 427-435.

4. CORREIA, D. et al. Uso do pó da casca de coco na formulação de substratos para formação de mudas enxertadas de cajueiro anão precoce. Revista Brasileira de Fruticultura, v. 25, n. 3, p. 557-558, 2003.

5. DAMASCO, O. P. et al. Gibberellic acid detection of dwarf off-types in micropropagated Cavendish bananas. Australian Journal of Experimental Agriculture, v. 36, n. 2, p. 237-241, 1996.

6. GROLLI, P. R. Composto de lixo domiciliar urbano como condicionador de substratos para plantas arbóreas. 1991. 126 p. Dissertação (Mestrado em Fitotecnia) - Universidade Federal do Rio Grande do Sul, Porto Alegre, 1991.

7. HUETT, D. O. Fertiliser use effciency by containerised nursery plants: 2. nutrient leaching. Australian Journal Agriculture Research, v. 48, n. 2, p. 259-265, 1997.

8. KOZLOWSKI, T. T.; KRAMER, P. J.; PALLARDY, S. G. The physiological ecology of woody plants. New York: Academic Press, 1991. 657 p.

9. OLIVEIRA, P. S. R.; GUALBERTO, R.; FAVORETO, A. J. Efeito do Osmocote adicionado ao substrato Rendimax na produção de mudas de café em tubetes. In: CONGRRESSO BRASILEIRO DE PESQUISAS CAFEEIRAS, 21., 1995, Caxambu. Anais... Caxambu: PROCAFE/DENAC, 1995, p. 70-72.

10. MACIEL, A. L. de R.; SILVA, A. B. da; PASQUAL, M. Aclimatação de plantas de violeta (Saintpaulia ionantha Wendl) obtidas in vitro: efeitos do substrato. Ciência e Agrotecnologia, v. 24, n. 1, p. 9-12, 2000.

11. MILLER, J. H.; JONES, N. Organic and compost-based growing media for tree seedlings nurseries. Washington: The World Bank, 1995. 88 p. (World Bank Technical Paper, 264).

12. MORGADO, I. F. Resíduos agroindustriais prensados como substrato para a produção de mudas de Eucalyptus grandis Hill ex Maiden e Saccharum spp. 1998. 102 f. Tese (Doutorado em Produção Vegetal) Universidade Estadual do Norte Fluminense, Campos dos Goytacazes, 1998.

13. MORGADO, I. F. et al. Resíduos agroindustriais prensados como substrato para a produção de mudas de cana-deaçúcar. Scientia Agricola, v. 57, n. 4, p. 709-712, 2000.

14. RESENDE, J. T. V. de. et al. Utilização de resíduos agroindustriais como substrato na produção de mudas de tomateiro. Ambiência, v. 1, n. 1, p. 25-29, 2005.

15. SCARANARI, C. Aclimatação de mudas micropropagadas de bananeira (Musa spp.) cv. Grande Naine. 2006. 126 f. Tese (Doutorado em Engenharia Agrícola) - Universidade Estadual de Campinas, Campinas, 2006.

16. SCIVITTARO, W. B.; OLIVEIRA, R. P. de, RADMANN, E. B. Doses de fertilizante de liberação lenta na formação do porta-enxerto 'Trifoliata. Revista Brasileira de Fruticultura, v. 26, n. 3, p. 520-523, 2004.

17. SEABRA FILHO, M. Efeito de composições e superfosfato simples no crescimento e nutrição de mudas de bananeira cv. Nanicão obtidas por propagação rápida "in vivo". 1994. 103 p. Dissertação (Mestrado em Fitotecnia). Escola Superior de Agricultura de Lavras, Universidade Federal de Lavras, Lavras, 1994.

18. SERRANO, L. A. L. et al. Utilização de substrato composto por resíduos da agroindústria canavieira para produção de mudas de maracujazeiro-amarelo. Revista Brasileira de Fruticultura, v. 28, n. 3, p. 487-491, 2006.

19. SHARMA, G. C. Controlled-release fertilizers and horticultural applications. Scientia Horticulturae, v. 11, n. 2, p. 107-129, 1979.

20. SILVA, C. R. R.; SOUTO, R. F.; MENEGUCCI, J. L. P. Propagação da bananeira. Informe Agropecuário, v. 20, n. 196, p. 16-20, 1999.

21. SOUSA, H. U. de. et al. Nutrição de mudas de bananeira em função de substratos e doses de superfosfato simples. Ciência e Agrotecnologia, v. 24, edição especial, p. 64-73, 2000.

22. VALE, L. S. do. et al. Efeito de diferentes misturas de substrato e tamanho de recipientes na produção de mudas de mamoeiro. In: ENCONTRO NACIONAL DE SUBSTRATOS, 4., 2004, Viçosa. Anais... Viçosa: UFV. 2004. p. 385. 